\title{
3-D SIMULATIONS OF KELVIN-HELMHOLTZ INSTABILITIES IN SUPERSONIC JETS
}

\author{
P. ROSSI, G. BODO, S. MASSAGLIA AND A. FERRARI \\ Osservatorio Astronomico di Torino \\ Strada dell'Osservatorio 20, 10025 Pino Torinese, Italy \\ AND \\ A. MALAGOLI \\ EFI/LASR, University of Chicago \\ 933 E.56 ${ }^{\text {th }}$, Chicago, IL 60637, USA
}

One of the key processes governing the structure and evolution of astrophysical jets is their interaction with the surrounding medium. A jet can deposit momentum and energy in the ambient medium, and entrain external material. The main physical process responsible for mixing between a jet flow and the ambient medium is the Kelvin-Helmholtz (KH) instability. We have previously analysed the $2 \mathrm{D}$ evolution of the axisymmetric modes of a cylindrical jet (Bodo et al 1994) and of the antisymmetric modes of a planar slab jet (Bodo et al 1995). These last are thought to give indications of the 3D evolution of the helical modes of a cylinder, since the linear behavior is very similar. In this contribution we present some preliminary results of fully $3 \mathrm{D}$ simulations comparing them with the mentioned $2 \mathrm{D}$ results.

The simulations have been performed using a PPM type code; the computational domain is covered by a uniform grid of $128 \times 128 \times 128$ points. In the initial configuration a cylindrical flow, with velocity $V_{0}$ directed along the $x$ direction of a cartesian coordinate system, is surrounded by a medium at rest. The jet radius $a$ encompasses 25 grid points and the computational domain extends in the $y$ and $z$ directions from $-2.56 a$ to $+2.56 a$. Periodic boundary conditions are used at the $x$ boundaries, while free outflow conditions are used at the $y$ and $z$ boundaries. A perturbation to the transverse velocity $v_{y}$ is superimposed to this equilibrium structure and the evolution is followed by the numerical calculation. Explicit detailed expressions for the equilibrium configuration and the form of the perturbation can be found in Bodo et al $(1994,1995)$. The system in characterised by two main 

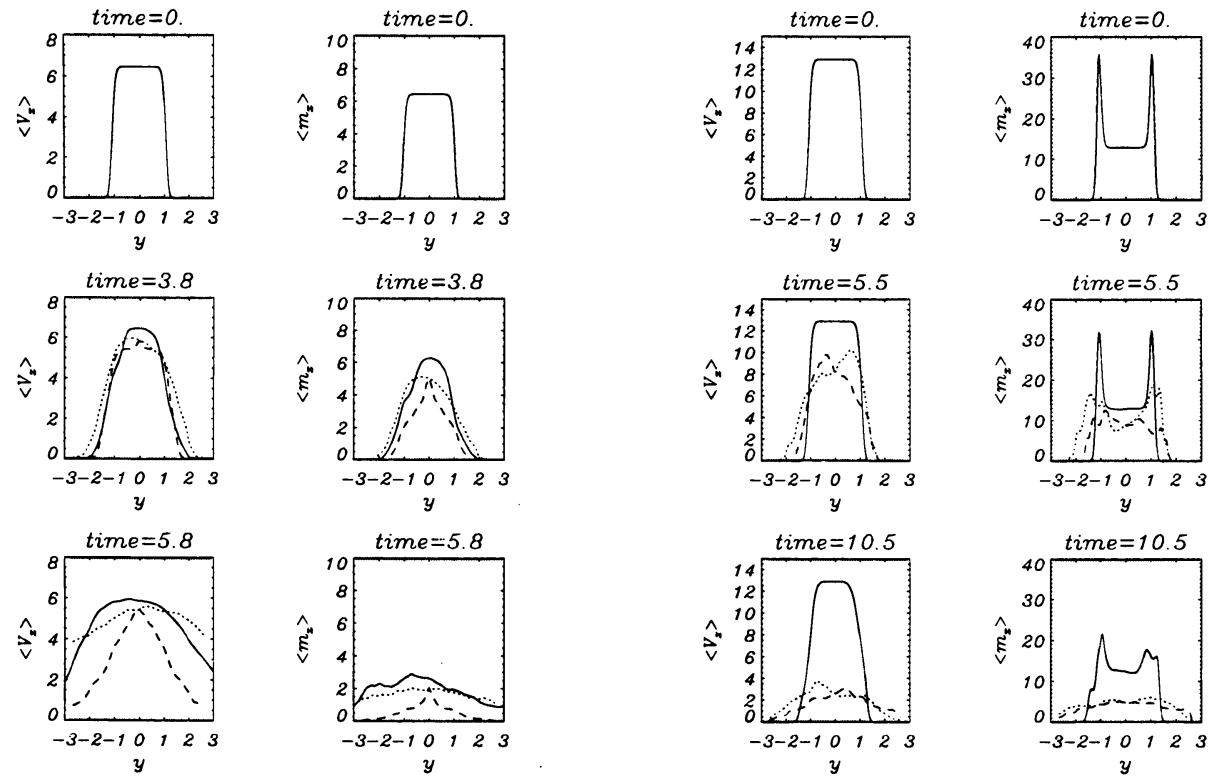

Figure 1. Comparison between the average distributions of the $x$ components of velocity and momentum for the $2 \mathrm{D}$ and $3 \mathrm{D}$ calculations. The solid line refers to the $2 \mathrm{D}$ case, while the dashed and dotted lines refer respectively to a $y$ and $z$ cut in the $3 \mathrm{D}$ data. The time is measured in units of the sound crossing time over the jet radius.

parameters: the Mach number $M$ of the flow (with respect to the internal sound speed) and the density ratio $\nu$ between external medium and jet. We have performed two simulations with $M=5, \nu=0.1$ (case A) and $M=10, \nu=10$ (case B) respectively, i.e. for a jet denser than the ambient medium (case $A$ ) and a jet less dense than the ambient medium (case B).

We have compared the distributions of longitudinal velocity and momentum averaged over the periodic $x$ directions with the analogous distributions obtained in the 2D planar slab calculation (Bodo et al 1995). From the figure we can see that, while the distribution are quite similar in case A, the evolution appears to be much faster in $3 \mathrm{D}$ for case $\mathrm{B}$.

\section{References}

Bodo, G., Massaglia, S., Ferrari, A. and Trussoni, E. (1994), $A \& A, 283,655$.

Bodo, G., Massaglia, S., Rossi, P., Ferrari, A., Rosner, R. and Malagoli A. (1995), $A \& A$, in press. 